\title{
Minimizing Energy Cost in Multi-Legged Walking Machines
}

\author{
Teresa Zielinska
}

Published online: 20 June 2016

(C) The Author(s) 2016. This article is published with open access at Springerlink.com

\begin{abstract}
Due to their ability to avoid obstacles and to move over difficult terrain, moreover having the ability to adjust their posture, walking machines for many years have been considered as very promising devices for inspection, exploration and surveyance tasks, however still they have not been widely applied. One of the main limitations is the power supply. Six legged walking machines are robust from the point of view of their walking stability in difficult terrain, but their actuators (18 if each leg has active 3 DOF's) adds to their weight what increases the energy consumption. The higher energy consumption requires more efficient batteries, but usually those are heavier, what again increases the energy demand. Therefore at the design stage a detailed analysis is required of how to decrease the energy consumption. This paper studies energy consumption considering the tripod gait of hexapods. The method used for energy evaluation is presented and the results are discussed. The discussion of energy saving both for the leg transfer phase and during the support phase, which is the most demanding phase, is presented. The energy consumption is expressed in
\end{abstract}

This work is supported by Warsaw University of Technology, FPAE Statutory Funds and EMARO.

T. Zielinska $(\bowtie)$

Institute of Aeronautics and Applied Mechanics

(WUT-IAAM), Warsaw University of Technology,

ul. Nowowiejska 24, 00-665 Warsaw, Poland

e-mail: teresaz@meil.pw.edu.pl the normalized form, depending on the normalized leg proportions, body height and step length. The straight line forward/backward and side walking are analyzed. The aim of the studies is to provide to the designers the information about favorable leg proportions taking into account the reduction of required energy and to provide the information which leg posture should be selected.

Keywords Walking machines · Energy optimal design · Energy favourable leg posture $\cdot$ Hexapods energy efficiency $\cdot$ Hexapods

\section{Introduction}

For many years multi-legged walking machines (machines with more than 2 legs) have been considered as appropriate devices for security, inspection and exploration tasks. In comparison with the wheeled robots they have better ability to move in unstructured environment, i.e. over uneven terrain. They can climb over obstacles and avoid ditches. They can adapt their posture and gait type accordingly. According to International Federation of Robotics (IFR) Statistical Department in 2012 the overall number of professional non-industrial robots approached 126000 units [6]. In 2011 and 2012 annual sale of such professional robots was about 15000 units. IFR reported that in 2012 the highest number (in units) of sold professional robots were the defence robots $-47 \%$, and other 
field robots $-41 \%$. The success of robots in military applications is underscored by the newest four-legged running robots developed for DARPA. Those robots were demonstrated for the first time in 2012. They compose the so called Legged Squad Support System (LS3) for assisting marines. Those robots can move semi-autonomously in difficult terrain transporting heavy loads. Robots designed for rescue operations, the robots for humanitarian demining and robots for space exploration act in hazardous environments. All the above mentioned robots carry their power supply (usually batteries) on board and should operate for a rather long time without recharging. The saving of energy is very important in this case. Success of LS3 confirms the expectations that walking machines having greater motion abilities are a good option in special applications. They are the emerging kind of professional robots. Unfortunately, despite of the work devoted to walking machines for search [29] and demining [5], many publications in the field of walking machines concerns the devices for laboratory research. The problem of energy savings is not treated there so often. In the early publications in this area relations between specific resistance (normalized energy) and the set of normalized walking machine parameters was investigated [28]. The studies produced suggestions concerning favorable relations between step length, body height, trunk and leg masses. In [15] five rules for selecting hexapod body height, motion velocity, footholds, the gait duty factor, and the step length were proposed, taking into account energy efficiency. The efficiency of wave gaits for hexapods was investigated in [3]. In [9] the studies of energy optimal four-legged robot gaits were presented. In [23, 24] the effect of gait parameters such as duty factor, walking speed and leg stroke on energy spending during turning motion was investigated. Authors come to the conclusion that the peaks of joint torques and leg end-forces in hexapod gait decrease when the duty factor of a sixlegged robot gait increases. In recent years the most advanced studies were presented in [25]. The problem of energy minimization for a given hexapod moving over irregular terrain was studied. The selection of the stepping patterns and leg-end trajectories for energy optimization were investigated taking into account the properties of DC motors. The same research group tested the relation between that hexapod leg posture and energy consumption concluding that energy saving is associated with insect type posture [26]. Work devoted to the motion efficiency depending on animal gait patterns and body structure has been also conducted by biologists $[1,4,10]$.

In this paper we study the relationship between the leg posture, leg proportions and energy consumption during the support phase for backward/forward straight line walking on horizontal and inclined surface, and for straight line side walking on horizontal surface. The work expands the result presented in [31], where only favorable leg link proportions from the point of view of the energy consumption were analyzed for straight line walking over a horizontal surface with the leg-end forces obtained using simplified method neglecting the motion dynamics. As the majority of researchers do, when developing the machines for real missions, we focus on hexapods, because their postural stability is less sensitive to the environmental conditions than that of quadrupeds [2, $7,13,16,18,20]$. Moreover, they can walk using many types of statically stable gaits. An interesting overview of such devices can be found in [17].

The paper is organized as follows. The means of energy reduction in leg transfer phase and leg support phase are presented. The discussion on such a leg design as to decrease the energy demand in the transfer phase is carried out. Next we focus on the energy needed in the support phase. The formulas used for energy calculation are given considering the supporting legs. The general relations applied to the energy evaluation taking into account the motion dynamics are given. The problem of leg end forces evaluation for the tripod gait is discussed. Following sections provide the results on leg proportions and posture analysis resulting in a decrease of the consumed energy. The favorable, from the point of view of energy consumption, postures and link proportions are indicated both for forward and side walking with constant speed. The final conclusions are drawn indicating the most appropriate leg postures.

\section{Decreasing Energy Demand in the Transfer Phase}

Multi-legged walking robots often use identical legs and so their contribution to body displacement is the same. This is unlike in the animals. The range of joint motion is often limited resulting in small legend work-space. It is a disadvantage, as a large leg 


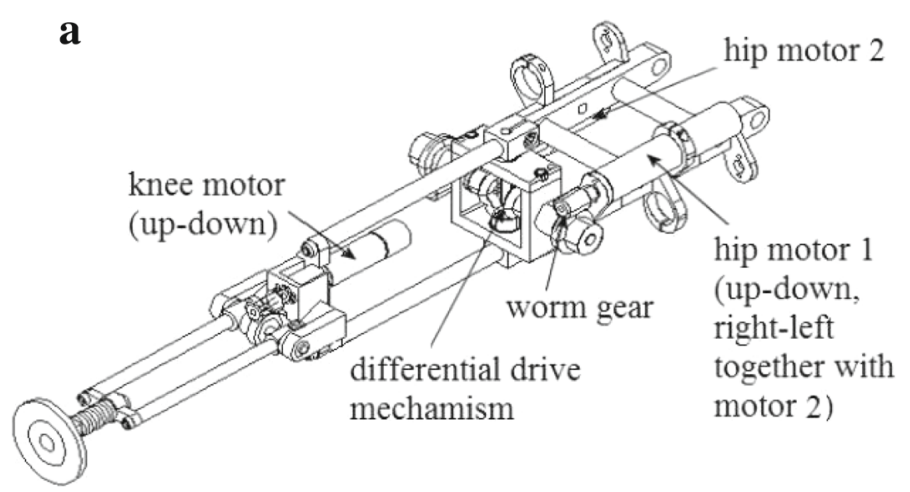

b
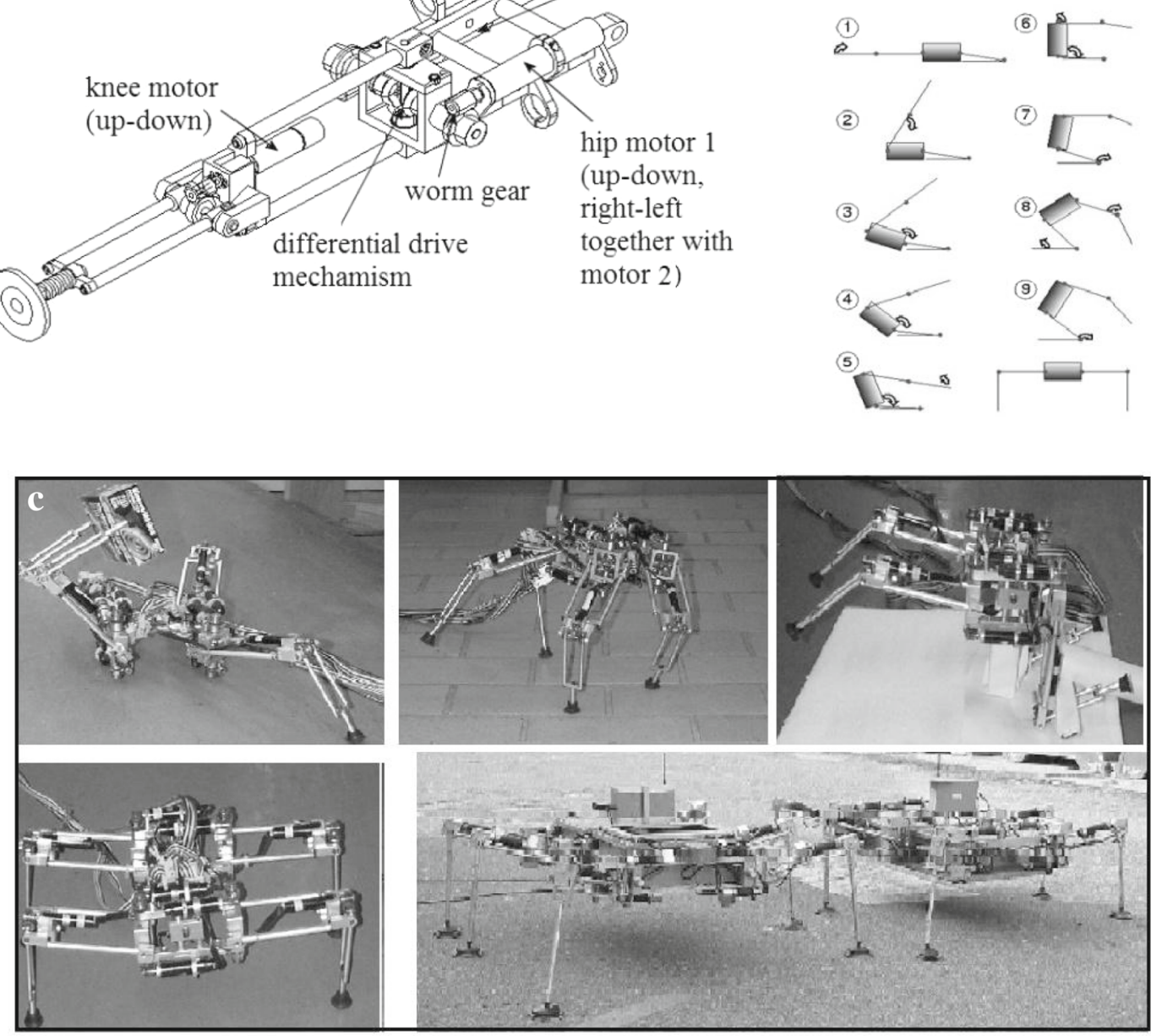

d

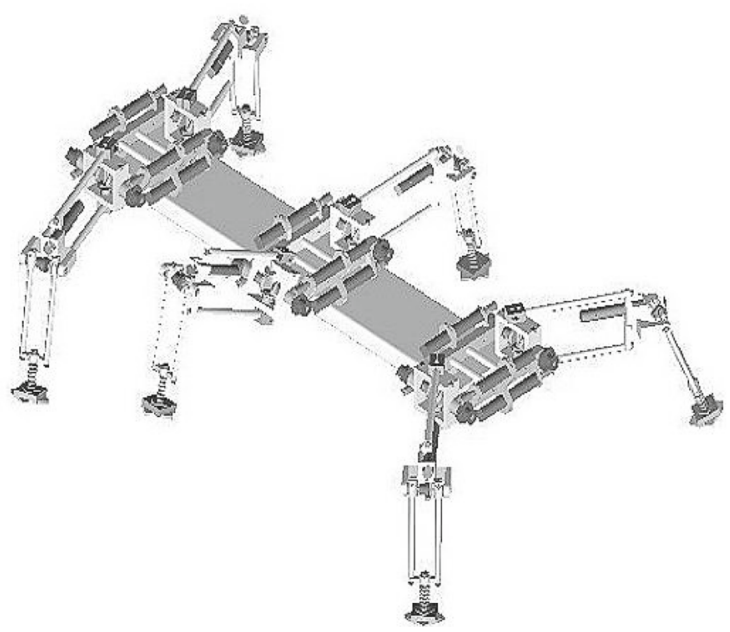

Fig. 1 The leg module developed by our team (a), the sequence of postures used for turn over motion (b), pictures of walking machines with modular legs - the quadrupeds illustrate different leg postures and large work space of the legs, - hexapods used in autonomous exploration (c), schematic view of the hexapod (d) 
work-space enables not only walking, but climbing, crawling and handling objects. Moreover, the legs with large motion range can be folded for transportation of the machine. The turn-over ability assured by a large work-space is important for robots carrying specialized equipment located in one part of the body, e.g. on the top of the trunk. Moreover, large workspace offers flexibility in adjusting the leg posture in order to decrease the consumed energy. Unfortunately not many robots offer such possibilities. Analyzing the design concepts used in hexapods it can be noticed that two degrees of freedom in the hip joint are separated, as the result only one motor is attached to the trunk and the second one is affixed to the thigh [5, 30]. In effect a typical three degrees of freedom leg [8, $12,19,30]$ must carry two motors (one hip motor plus one knee motor) during the transfer phases. Reducing the number of motors attached to a leg significantly decreases the energy consumed during transfer phases. It is very important, because over the walking distance the amount of transfer phases is significant. For example, assuming the step length equal to $0.5 \mathrm{~m}$, the number of transfer phases for the walking distance of $1 \mathrm{~km}$ covered using a tripod gait is 6000 . If each leg will carry not 2 but one motor it will significantly reduce the consumed energy.

One of the design concepts reducing the weight of the leg uses modular design (designer J.Heng) [32].
Each leg together with its actuators constitutes a separate module which can be easily attached to the trunk with two motors fixed to it. Differential mechanism in the hip joint assures coupled two degrees of freedom. Only the knee motor is carried out during the leg transfer. The two hip motors together actuate the most loaded hip joint. Such design was successfully applied in the family of walking machines (Fig. 1) developed by our team, starting from the small size and light quadrupeds: $15 \mathrm{~cm}$ height and $2 \mathrm{~kg}$ mass, and ending with hexapods for exploration [29]: $60 \mathrm{~cm}$ height and $40 \mathrm{~kg}$ mass with a payload $40 \mathrm{kG}$. As it is illustrated, the legs can be easily adjusted to different postures. They can also work as manipulators and they support the turn-over motion - what is a significant advantage for robots involved in rescue missions.

\section{Analyzing Energy Demand - Support Phase}

The most energy consuming motion phase is the support phase when the legs support the weight of the machine and push it. Leg transfer is an obvious requirement for stepping, however the energy consumption is here significantly smaller. When selecting the motors for a walking machine the needed motor torques during energy demanding support phase and the required angular speed for velocity demanding
Fig. 2 Block diagram illustrating the sequence of actions performed for energy evaluation

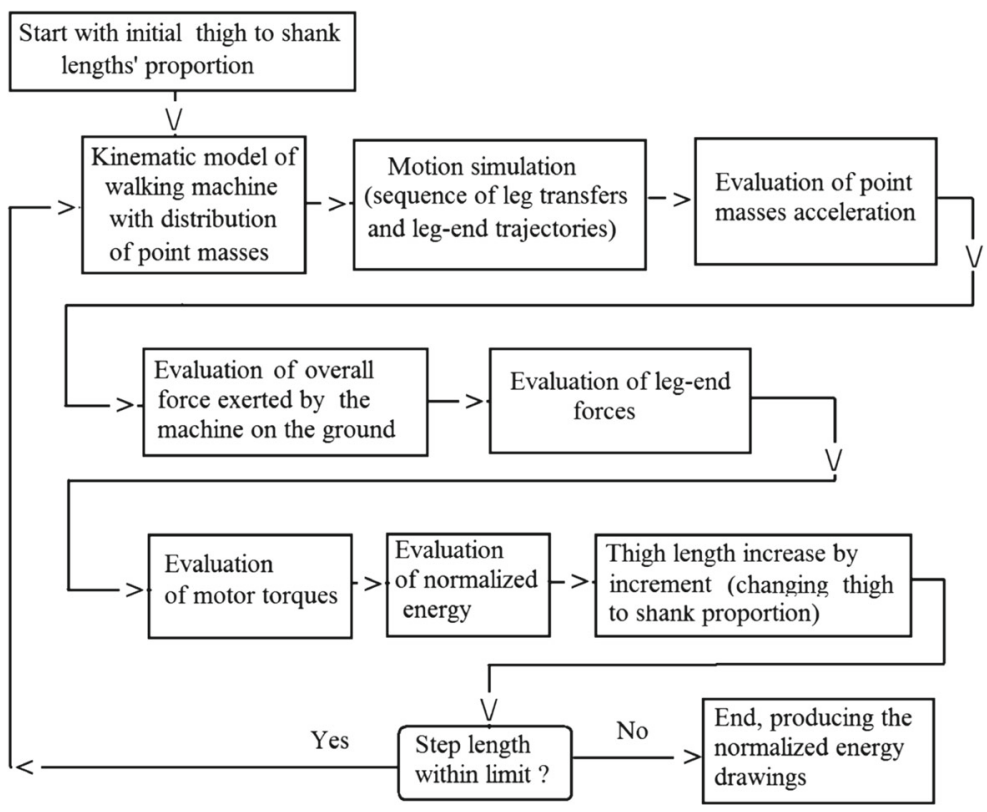


transfer phase [30] are considered. Adequate design of the leg-end trajectory during leg transfer reduces the range of angular velocities and accelerations and decreases jerks [32]. The selection of actuators should take into account that it is important not to oversize the knee motor, because it increases the leg weight without reason. On the other hand the motor power must guarantee that attainable torque will enable the execution of all the required gaits with the expected machine payload.

The presented in this section method of energy evaluation can be applied for any statically stable walking machine. Figure 2 shows the block diagram of the whole procedure. The knowledge of leg-end forces is needed here, therefore for evaluation of inertial forces the structure of the machine and the gait must be defined. In consequence to our aim we shall focus on hexapods moving using tripod gait. The legs with two degrees of freedom in the hip joint and one degree of freedom in the knee will be considered in calculations.

\subsection{Energy Consumption in the Support Phase}

Actuating torque is calculated using the dynamics equation:

$\Gamma_{l}=M_{l}(\theta) \ddot{\theta}+C_{l}(\theta, \dot{\theta})+G_{l}(\theta)$

for the transferred leg, and

$$
\Gamma_{l}=M_{l}(\theta) \ddot{\theta}+C_{l}(\theta, \dot{\theta})+G_{l}(\theta)+J_{l}^{T} F_{l}
$$

for the supporting leg, where: $\Gamma_{1}$ is an $n \times 1$ vector of actuating torques, $n$ is the number of actuated DOFs, $\mathbf{M}_{\mathbf{l}}(\theta)$ is an $n \times n$ inertia matrix, $\mathbf{C}_{l}(\theta, \dot{\theta})$ is an $n \times 1$ vector of centrifugal and Coriolis terms, $\mathbf{G}_{\mathbf{l}}(\theta)$ is an $n \times 1$ vector of gravitation terms, $\theta, \dot{\theta}, \ddot{\theta}$ are the joint angles, angular velocities and accelerations, $\mathbf{J}_{\mathbf{l}}^{\mathbf{T}}$ is an $n \times 3$ transposed jacobian matrix and $\mathbf{F}_{\mathbf{l}}$ is a $3 \times 1$ vector of ground contact forces.

Analyzing energy consumption in the support phase, we shall neglect in rel. (2) the first three terms. For walking machines moving with relatively low speeds with almost constant leg posture during the support phase and with lightweight legs, this part is much smaller than the last component. Our studies showed that this part is responsible for no more than $10 \%$ of the required torque [32].
Taking into account the above, the vector of leg joint torques is expressed by:

$\boldsymbol{\Gamma}_{l}=J_{l}(\theta)^{T} F_{l}, \quad \boldsymbol{\Gamma}_{l}=\left[\tau_{1}, \ldots . . \tau_{i}, . ., \tau_{N}\right]^{T}$

If the motor torque is $\tau_{i}(i=1, \ldots, N$, where $N-$ is the total number of actuated joints) and the needed angular velocity is equal to $\dot{\theta}_{i}$ then the motor power $P$ is:

$P=\tau_{i} \dot{\theta}_{i}$

The power integrated over the walking time gives the consumed (needed) energy $E$ :

$E=\int_{0}^{T_{f}} P d t$

\subsection{General Relations}

Leg-end forces are calculated using force and torque equilibrium conditions. For the machine moving with constant speed the force equilibrium condition is expressed by:

$$
\sum_{\mathbf{l p}} \mathbf{F}_{\mathbf{l} \mathbf{p}}=\left(m_{b}+\sum_{l} \sum_{i} m_{l i}\right) \mathbf{G}_{\mathbf{g}}+\sum_{l} \sum_{i} m_{l i} \mathbf{A}_{l i}
$$

$\sum_{\mathbf{l p}} \mathbf{F}_{\mathbf{l p}}=\left[f_{\mathbf{l p} x}, f_{\mathbf{l p} y}, f_{\mathbf{l p} z}\right]^{T}$ is the sum of leg-end forces $(l$ is the leg index and $\mathbf{l p}$ indicates the leg in the support phase $), \mathbf{G}_{\mathbf{g}}=[0,0, g]^{T}(g-$ gravitational acceleration), $\mathbf{A}_{l i}=\left[a_{l i x}, a_{l i y}, a_{l i z}\right]^{T}-$ accelerations of the leg segment point masses, $m_{b}$ is the trunk mass (without legs), $m_{l i}$ is the mass of the $i$-th link of the $l$-th leg.

For walking machines we use simplified moments equilibrium condition, neglecting the inertial effects due to the revolution of the machine's parts:

$\sum_{\mathbf{l p}}\left(\mathbf{r}_{l \mathbf{p}}-\mathbf{r}_{\mathbf{a}}\right) \times \mathbf{F}_{l \mathbf{p}}=0$

$\mathbf{r}_{\mathbf{l p}}$ is the vector connecting the leg-end to the origin of the non-moving reference frame, $\mathbf{r}_{\mathbf{a}}$ is the vector from the machine center of mass to the origin of the reference frame (Fig. 3):

$\mathbf{r}_{\mathbf{a}}=\frac{m_{b} \mathbf{r}+\sum_{l} \sum_{i} m_{l i} \mathbf{r}_{l i}}{m_{b}+\sum_{l} \sum_{i} m_{l i}}$ 


\subsection{Leg-End Forces}

For the assumed machine parameters we evaluated the distribution of leg-end forces using Eqs. 6 and 7. Next the torques were calculated using relation (3) with the jacobian calculated taking into account (21). Finally, Eqs. 4 and 5 were applied producing the needed energy.

As our aim was to deliver the guidelines for the leg designers, therefore we have not considered the energy consumption due to speed changes and terrain unevenness. By remote operation the machine first walks to the area to be searched. For this part of the mission the energy saving is most important. In the main part of the mission the priority is to fulfill the task (e.g. the identification of the searched object) therefore the energy is of secondary importance. In that part of operation the machine must adjust the posture according to the mission aim and energy saving is not a priority. The last part - return to the base station is not so critical taking into account the energy, the fault due to lack of power supply should not affect the mission core. Focusing on the need for energy savings during the first part of the mission, the motion with constant speed over horizontal surface was analyzed. It should be added that the access to the mission area usually is over a prepared path, what is not the case for the mission execution. A good example of such conditions is a demining operation.

In our studies only the part of the energy which is consumed by the legs supporting and pushing the body was taken into account. The energy needed for leg transfer is much lower. The leg segments are made of lightweight materials (e.g. composite material with appropriate weight to strength ratio). The motor with motion transmission system is located in the knee joint what results in mass concentration, therefore we approximated the mass of leg by one point mass located in the knee. The point mass of the trunk was located in its geometrical center (to get the proper balance the designers of walking machines distribute all internal equipment uniformly therefore such assumption conforms to reality) - Fig. 4 .

Without loosing generality we analysed a tripod gait. In comparison with the other possible gaits the supporting legs are most loaded in this case. We did not evaluate the energy for the transferred legs, however we have considered the motion of all point masses for those legs. For this purpose the simple rectangular shape of leg-end trajectory during transfer was assumed. Instantaneous positions of point masses were used when evaluating center of mass - rel. (8), and the inertial forces due to their motion were used when evaluating the leg-end forces. The forces exerted by three supporting legs on the ground during the tripod gait are equal to the gravity force acting on the machine and the inertial forces. Those forces are evaluated considering the point mass accelerations with respect to the non-moving reference frame. According to our assumption the machine moves with constant speed, the main body (trunk) does not undergo acceleration, therefore the inertial forces are obtained considering the accelerations in the reference frame attached to the body (Fig. 3):

$$
\begin{aligned}
F x= & \sum_{i=1}^{3} F x_{i p}=\sum_{i=1}^{3} \sum_{l=1}^{2} m_{l i} \ddot{x}_{l i} \\
F y= & \sum_{i=1}^{3} F y_{i p}=\sum_{i=1}^{3} \sum_{l=1}^{2} m_{l i} \ddot{y}_{l i} \\
F z= & \sum_{i=1}^{3} F z_{i p}=\sum_{i=1}^{3} \sum_{l=1}^{2} m_{l i} \ddot{z}_{l i} \\
& +\sum_{i=1}^{3} \sum_{l=1}^{2} m_{l i} g+m_{b} g
\end{aligned}
$$

In a stable posture the distribution of the leg-end forces is such that the roll and pitch moments $M_{x}$ and $M_{y}$ (8) acting on the COM are equilibrated. In postural stability analysis the moment $M_{z}$ causing the rotation around the vertical axis is neglected, because it is equilibrated due to friction [22,27]. Let us denote by $x_{\text {com }}, y_{\text {com }}, z_{\text {com }}$ - the machine's center of mass in the body reference frame (Fig. 3), where: $h$ - the distance from the supporting leg-ends to the origin of reference frame along the $Z$ axis (height of the machine), and by $x_{i p}, y_{i p}, z_{i p}, \quad i=1,2,3$, supporting leg-end coordinates with respect to the body frame (Fig. 3). According to Eq. 8 the moments are:

$$
\begin{aligned}
M_{x}= & \left(y_{1 p}-y_{c o m}\right) F z_{1 p}+\left(y_{2 p}-y_{c o m}\right) F z_{2 p} \\
& +\left(y_{3 p}-y_{c o m}\right) F z_{3 p}-\left(h-z_{c o m}\right) F x=0 \\
M_{y}= & -\left(x_{1 p}-x_{c o m}\right) F z_{1 p}+\left(x_{2 p}-x_{c o m}\right) F z_{2 p} \\
& +\left(x_{3 p}-x_{c o m}\right) F z_{3 p}-\left(h-z_{z c o m}\right) F y=0
\end{aligned}
$$


Fig. 3 Leg angle notation (a), coordinate frames (b), denotation of legs: RF right front, $\mathrm{RM}$ - right middle, $\mathrm{RH}$ - right hind, LF

- left front, LM - left middle, LH - left hind (c)



b $\begin{aligned} & \text { motion } \\ & \text { direction }\end{aligned}$

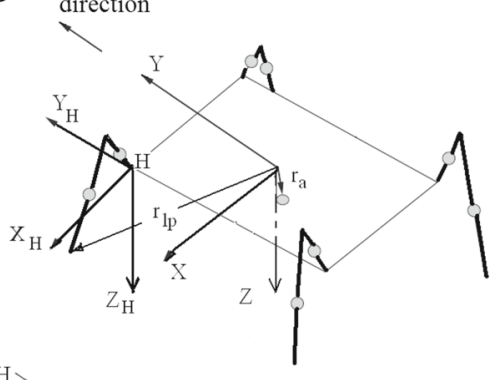

To abbreviate the notation we introduce: $\bar{x}_{1 p}=$ $x_{1 p}-x_{c o m}, \bar{y}_{1 p}=y_{1 p}-y_{c o m}, \bar{h}=h-z_{c o m}$. Using Eqs. 10 and 9 the vertical components of leg-end forces are obtained:

$$
\begin{aligned}
F z_{2 p} & =\frac{\left[\left(\bar{y}_{3 p} a+\bar{x}_{3 p} b\right] F z-b \bar{h} F x-a \bar{h} F y\right.}{\left(\bar{x}_{3 p}-\bar{x}_{2 p}\right) b-a\left(\bar{y}_{2 p}-\bar{y}_{3 p}\right)} \\
F z_{1 p} & =\frac{\bar{h} F y-\bar{y}_{3 p} F z-\left(\bar{y}_{2 p}-\bar{y}_{3 p}\right) F z_{2 p}}{b} \\
F z_{3 p} & =F z-F z_{2 p}-F z_{1 p}
\end{aligned}
$$

where $a=\bar{x}_{3 p}-\bar{x}_{1 p}, b=\bar{y}_{1 p}-\bar{y}_{3 p}$.
The evaluation of horizontal components is more troublesome. The equation for the moment $M_{z}$ is not sufficient to obtain it, moreover the moment $M_{z}$ during walking is not reduced to zero. The friction phenomenon compensates the effect of $M_{z}$ if the horizontal forces do not exceed the boundary value for the slip to occur. The vertical components of the reaction forces are much higher than the horizontal ones. In the energy studies only vertical forces are often considered when evaluating the required motor torques. Neglecting the horizontal components produces no significant difference to the selection of the
Fig. 4 Outer and inner view of the trunk (a), (c), considered point mass distribution (b)






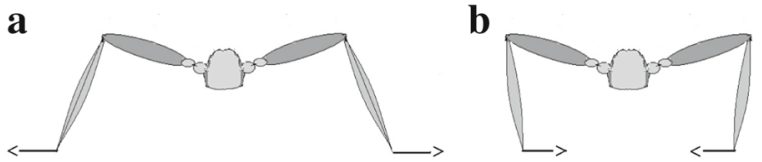

Fig. 5 Legs stretching the ground (a) and grasping it (b)

motors, however such simplification can adversely influence the energy consumption computations over longer time. The methods of the evaluation of all legend force components in general apply the forces and torques equilibrium conditions but there is the problem of not enough equations (for tripod gait there are 5 equations (9), (10) and 9 unknown force components). This problem is overcome using mathematical methods such as nonlinear programming [14], other optimization techniques, or the methods focusing more on the physics of gait such as the methods zeroing the forces in leg-pairs [21], but there is no universal approach.

Walking machines are non-linear systems, but due to lack of possibility of modeling the features of ground contact (especially in non-uniform natural terrain and with multiple closed kinematic chains created by the ground and legs contacting it) in practice the simplest position control is usually applied. In this case the same leg-end position can be obtained with slightly different joint torques, as long as the resultant horizontal forces will stay within friction cones the ,inconsistences" in the joint torques will be not visible. But this causes additional and not needed energy spending. This effect can be reduced in realtime by combining the leg-end position control with force control.

Having in mind the above we decided to evaluate and consider the horizontal force components in the energy studies too. We introduced the approach which is close to the physics of walking. The leg-end force along the motion direction ( $F y_{i p}$ for the forward motion and $F x_{i p}$ for side walking) were obtained assuming the value of this force for each leg keeps the same proportion as the value of vertical force, i.e.:

$F x y_{i p}=\frac{F z_{i p}}{F z} F x y$

where $F x y_{i p}, F x y$ is accordingly $F x_{i p}, F x$ or $F y_{i p}$, $F y$. Such an approach splits the total force into the smallest possible (in the sense of absolute values) components, and the legs which are bearing higher load develop higher tractive force. Evaluating the side components $F x_{i p}$ for straight line motion we considered the posture of the shank following the idea presented in [11]. If the shanks form with the ground level sharp angles it means that the legs stretch the ground - the $F x_{i p}$ components are oriented away from the body, otherwise the legs grasp the ground the $F x_{i p}$ components are oriented towards the body (Fig. 5). The methods of assigning the forces to the legs depends on the sign of the overall $F x$ component and on the number of legs which support each side of the body.

Case 1: body supported by one leg ( $1 p)$ on the right side and two legs $(2 p, 3 p)$ on the left side of the body

\section{For positive $F x$ :}

- when $\theta_{2}+\theta_{3} \geq 90^{\circ}$ the legs grasp the ground and:

$$
\begin{aligned}
& F x_{1 p}=F x-F x_{2 p}-F x_{3 p} \\
& F x_{2 p}=-\frac{F z_{2 p}}{F z} F x \\
& F x_{3 p}=-\frac{F z_{3 p}}{F z} F x
\end{aligned}
$$

- when $\theta_{2}+\theta_{3}<90^{\circ}$ legs are stretch the ground and:

$$
\begin{aligned}
F x_{1 p} & =-\frac{F z_{1 p}}{F z} F x \\
F x_{2 p} & =\frac{F z_{2 p}}{F z_{2 p}+F z_{3 p}}\left(F x-F x_{1 p}\right) \\
F x_{3 p} & =\frac{F z_{3 p}}{F z_{2 p}+F z_{3 p}}\left(F x-F x_{1 p}\right)
\end{aligned}
$$

For negative $F x$ :

- when $\theta_{2}+\theta_{3} \geq 90^{\circ}$ :

$$
\begin{aligned}
F x_{1 p} & =-\frac{F z_{1 p}}{F z} F x \\
F x_{2 p} & =\frac{F z_{2 p}}{F z_{2 p}+F z_{3 p}}\left(F x-F x_{1 p}\right) \\
F x_{3 p} & =\frac{F z_{3 p}}{F z_{2 p}+F z_{3 p}}\left(F x-F x_{1 p}\right)
\end{aligned}
$$


a


$F_{Z}$

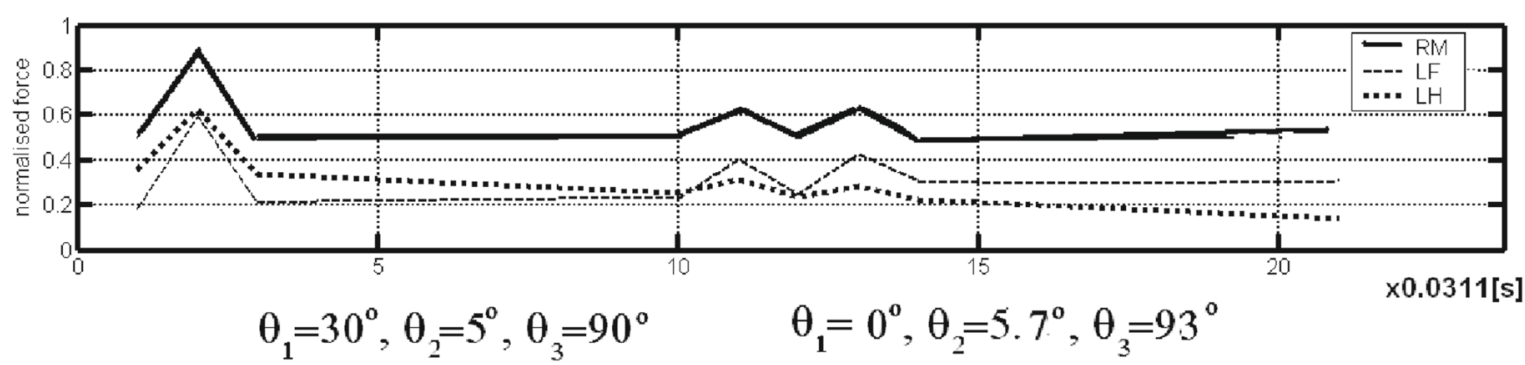

b
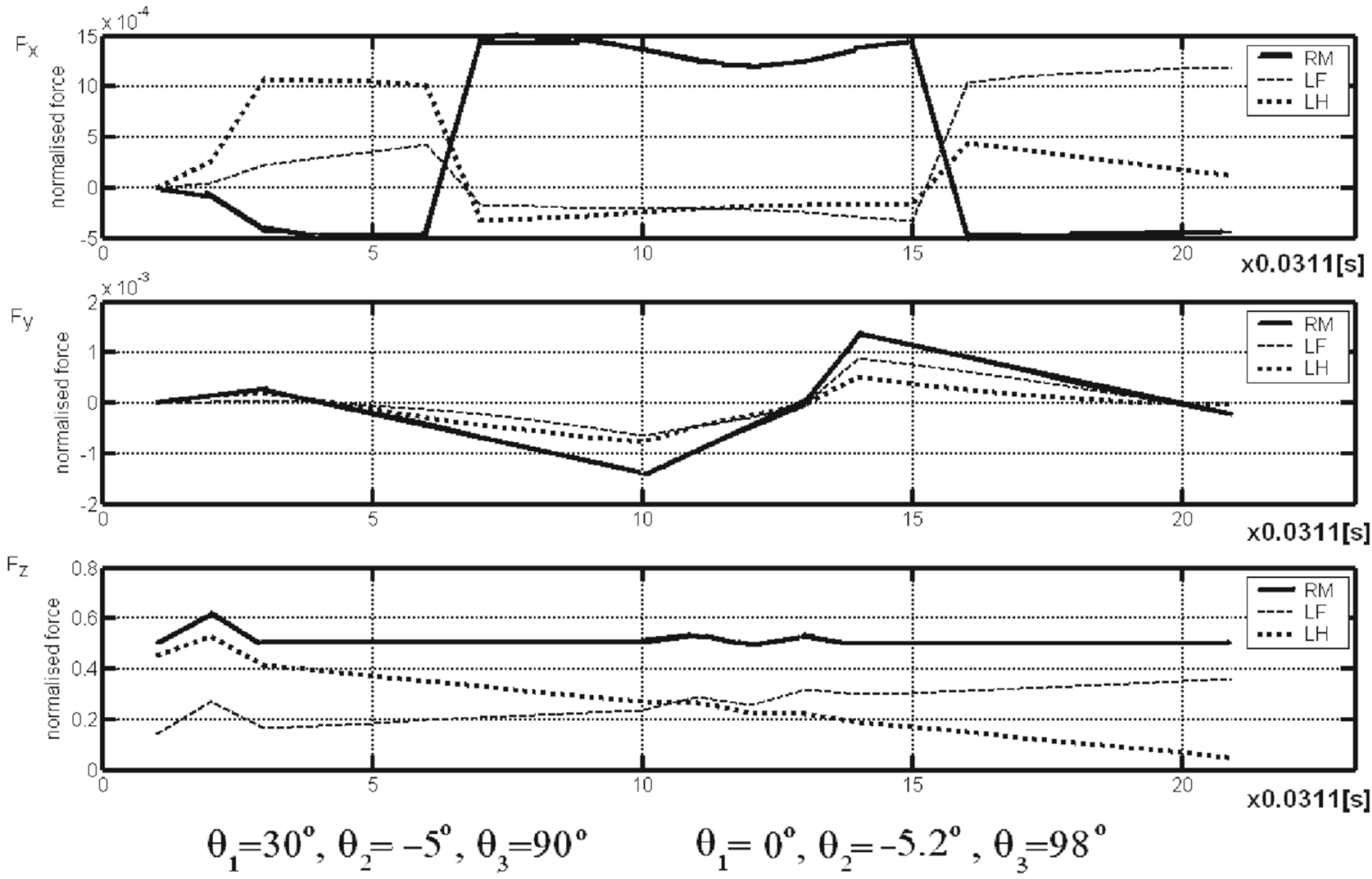

Fig. 6 Leg-end forces depend on posture: $\mathbf{a}$ - posture in the beginning of a step $\theta_{1}=30^{\circ}, \theta_{2}=5^{\circ}, \theta_{3}=90^{\circ}\left(\theta_{1}=0^{\circ}\right.$, $\theta_{2}=5.7^{\circ}, \theta_{3}=93^{\circ}$ in the middle of a step), $\mathbf{b}$ - posture in the beginning of a step $\theta_{1}=30^{\circ}, \theta_{2}=-5^{\circ}, \theta_{3}=90^{\circ}\left(\theta_{1}=0^{\circ}\right.$, $\theta_{2}=-5.2^{\circ}, \theta_{3}=98^{\circ}$ in the middle) 
- when $\theta_{2}+\theta_{3}<90^{\circ}$ :

$$
\begin{aligned}
& F x_{1 p}=F x-F x_{2 p}-F x_{3 p} \\
& F x_{2 p}=-\frac{F z_{2 p}}{F z} F x \\
& F x_{3 p}=-\frac{F z_{3 p}}{F z} F x
\end{aligned}
$$

Case 2: body supported by two legs on the right side $(2 p, 3 p)$ and one leg $(1 p)$ on the left side of the body

For positive $F x$ :

- when $\theta_{2}+\theta_{3} \geq 90^{\circ}$ the legs grasp the ground and:

$$
\begin{aligned}
F x_{1 p} & =-\frac{F z_{1 p}}{F z} F x \\
F x_{2 p} & =\frac{F z_{2 p}}{F z_{2 p}+F z_{3 p}}\left(F x-F x_{1 p}\right) \\
F x_{3 p} & =\frac{F z_{3 p}}{F z_{2 p}+F z_{3 p}}\left(F x-F x_{1 p}\right)
\end{aligned}
$$

- when $\theta_{2}+\theta_{3}<90^{\circ}$ legs stretch the ground and:

$$
\begin{aligned}
& F x_{1 p}=F x-F x_{2 p}-F x_{3 p} \\
& F x_{2 p}=-\frac{F z_{2 p}}{F z} F x \\
& F x_{3 p}=-\frac{F z_{3 p}}{F z} F x
\end{aligned}
$$

For negative $F x$ :

- when $\theta_{2}+\theta_{3} \geq 90^{\circ}$ :

$$
\begin{aligned}
& F x_{1 p}=F x-F x_{2 p}-F x_{3 p} \\
& F x_{2 p}=-\frac{F z_{2 p}}{F z} F x \\
& F x_{3 p}=-\frac{F z_{3 p}}{F z} F x
\end{aligned}
$$

- when $\theta_{2}+\theta_{3}<90^{\circ}$ :

$$
\begin{aligned}
F x_{1 p} & =-\frac{F z_{1 p}}{F z} F x \\
F x_{2 p} & =\frac{F z_{2 p}}{F z_{2 p}+F z_{3 p}}\left(F x-F x_{1 p}\right) \\
F x_{3 p} & =\frac{F z_{3 p}}{F z_{2 p}+F z_{3 p}}\left(F x-F x_{1 p}\right)
\end{aligned}
$$

Figure 6 shows leg-end forces depending on posture. The support triangle is created by RM, LF and $\mathrm{LH}$ legs. In Fig. 6a during the whole support phase the posture grasping the ground is maintained, each leg
$F x$ force maintains a constant sign. In Fig. 6b steps begin and end by stretching the ground and in its middle part the ground is grasped, the leg $F x$ forces change the signs accordingly.

\subsection{Results}

Typical leg kinematics $[8,12,30]$ illustrated in Fig. 3 were considered. In order to obtain the Jacobian needed in rel. (3) the expressions for leg-end coordinates must be used, in this case: [32]:

$$
\begin{aligned}
& x^{H}=l_{1} c_{1} c_{2}+l_{2} c_{1} c_{23} \\
& y^{H}=l_{1} s_{1} c_{2}+l_{2} s_{1} c_{23} \\
& z^{H}=l_{1} s_{2}+l_{2} s_{23}
\end{aligned}
$$

where $l_{1}$ is the length of thigh and $l_{2}$ is the length of the shank, and $s_{j}, c_{j}, c_{j k}$ are the sines and cosines of the $j$-th joint angle (Fig. 3a), or the sum of the angles $j$ and $k$.

The set of insect and reptile type leg postures was analyzed (Fig. 7). The trunk width to length ratio was 0.625 , the mass of the whole leg was equal to $9 \%$ of the trunk mass, what can be considered as a proper quantity for the machine transporting loads. The change of those proportions influences the amount of consumed energy, but it does not change the overall result concerning the advisable leg proportions and leg posture. In our investigations the second link of the leg (shank) had a constant length equal to half of the body length, the initial length of the first link (thigh) was smaller than the length of the shank and was increased in the test iterations. The possibly long thigh assures long step and large workspace. With longer steps the number of leg transfers over a given distance is lower - this reduces the energy spent on transferring legs. It should be noted that those transfers do not propel the machine, but consume power. The sufficient leg-end workspace is important a

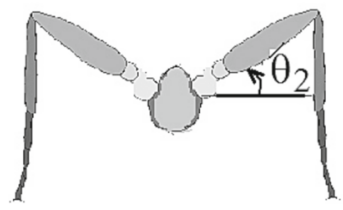

b

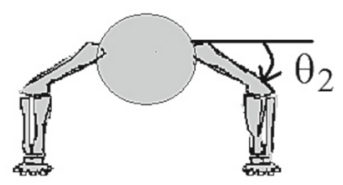

Fig. 7 Leg postures: insect type with negative $\theta_{2}-\mathbf{a}$, reptile type with positive $\theta_{2}-\mathbf{b}$ 

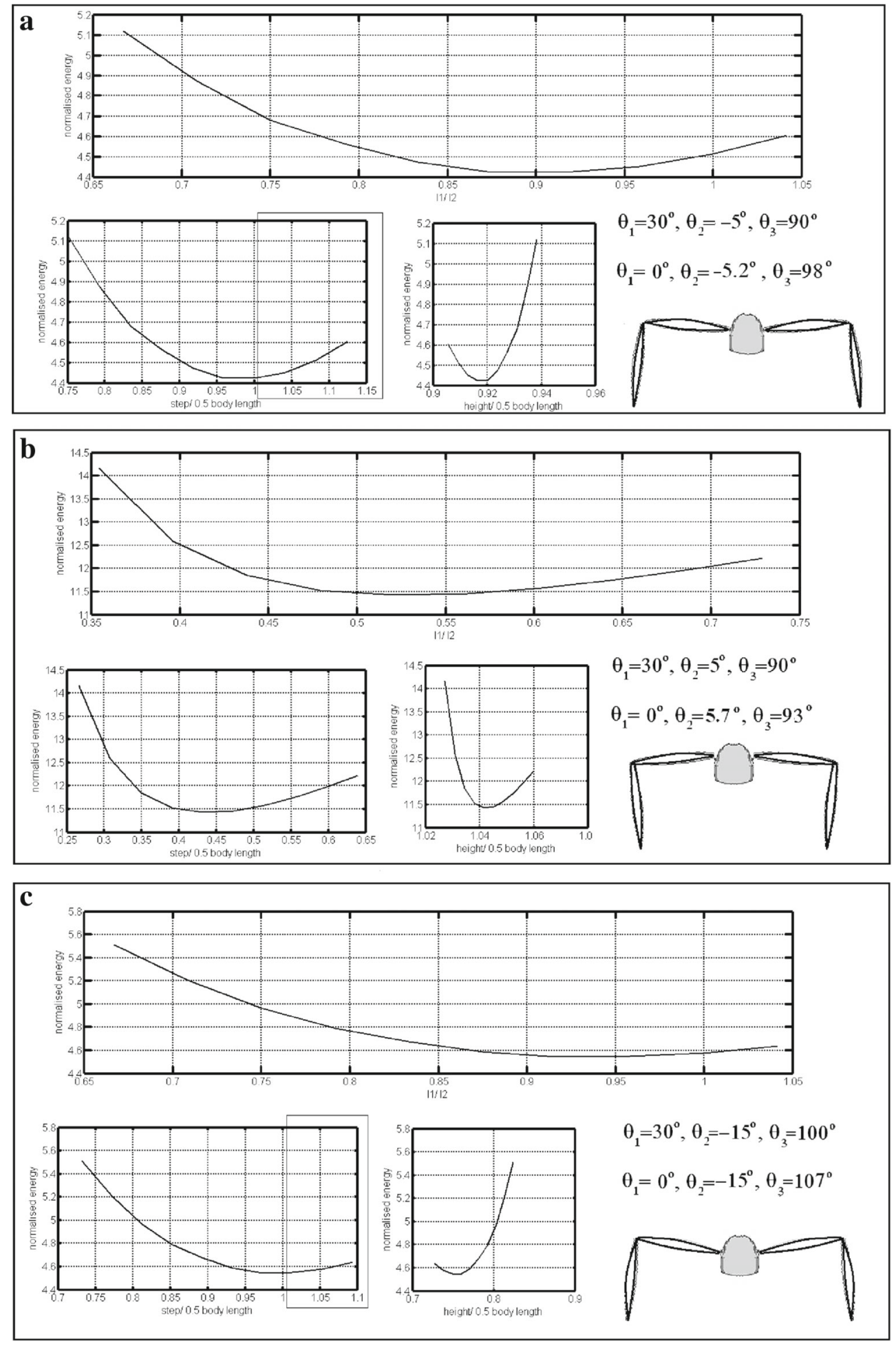

Fig. 8 Results for straight line walking: a - posture $\theta_{1}=30^{\circ}$, $\theta_{2}=-5^{\circ}, \theta_{3}=90^{\circ}$ in the beginning of the support phase $\left(\theta_{1}=0^{\circ}, \theta_{2}=-5^{\circ}, \theta_{3}=90^{\circ}\right.$ in the middle $), \mathbf{b}$ - posture $\theta_{1}=30^{\circ}, \theta_{2}=5^{\circ}, \theta_{3}=90^{\circ}$ in the beginning of the support phase $\left(\theta_{1}=0^{\circ}, \theta_{2}=-5.7^{\circ}, \theta_{3}=93^{\circ}\right.$ in the middle $), \mathbf{c}-$ posture $\theta_{1}=30^{\circ}, \theta_{2}=-15^{\circ}, \theta_{3}=100^{\circ}$ in the beginning of the support phase $\left(\theta_{1}=0^{\circ}, \theta_{2}=-15^{\circ}, \theta_{3}=107^{\circ}\right.$ in the middle) 

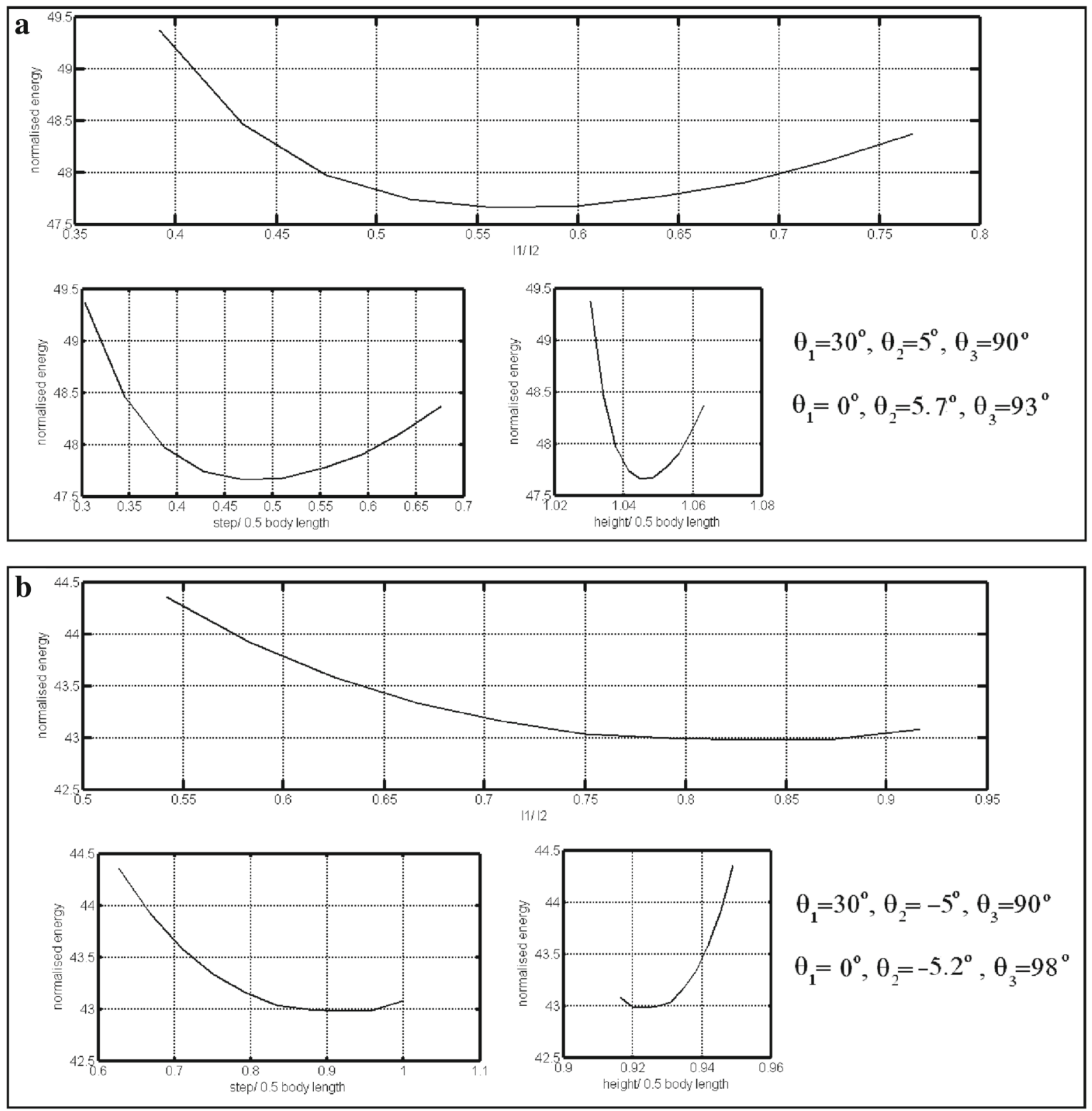

Fig. 9 Results for uphill walking. a - posture $\theta_{1}=30^{\circ}, \theta_{2}=$ $5^{\circ}, \theta_{3}=90^{\circ}$ in the beginning of the support phase $\left(\theta_{1}=0^{\circ}\right.$, $\theta_{2}=5.7^{\circ}, \theta_{3}=93^{\circ}$ in the middle $), \mathbf{b}-$ posture $\theta_{1}=30^{\circ}$,

for manoeuvres and when the legs are used as manipulators (note that the human arm and fore-arm have comparable length). It was assumed that during each step the angular position $\theta_{2}$ for maximum protraction and retraction are the same and equal to $30^{\circ}$ and $-30^{\circ}$ respectively. With such a range the step is possibly long. The motion speed was $3 \mathrm{~km} / \mathrm{h}$. For generality the $\theta_{2}=-5^{\circ}, \theta_{3}=90^{\circ}$ in the beginning of the support phase $\left(\theta_{1}=0^{\circ}, \theta_{2}=-5.2^{\circ}, \theta_{3}=98^{\circ}\right.$ in the middle $)$

step length and body height was normalized to half of the body length. The obtained energy was divided by the traveled distance multiplied by the machine's weight what gave specific resistance. According to the used terminology the specific resistance is the energy needed for carrying of a unit weight over a unit distance [28]. 

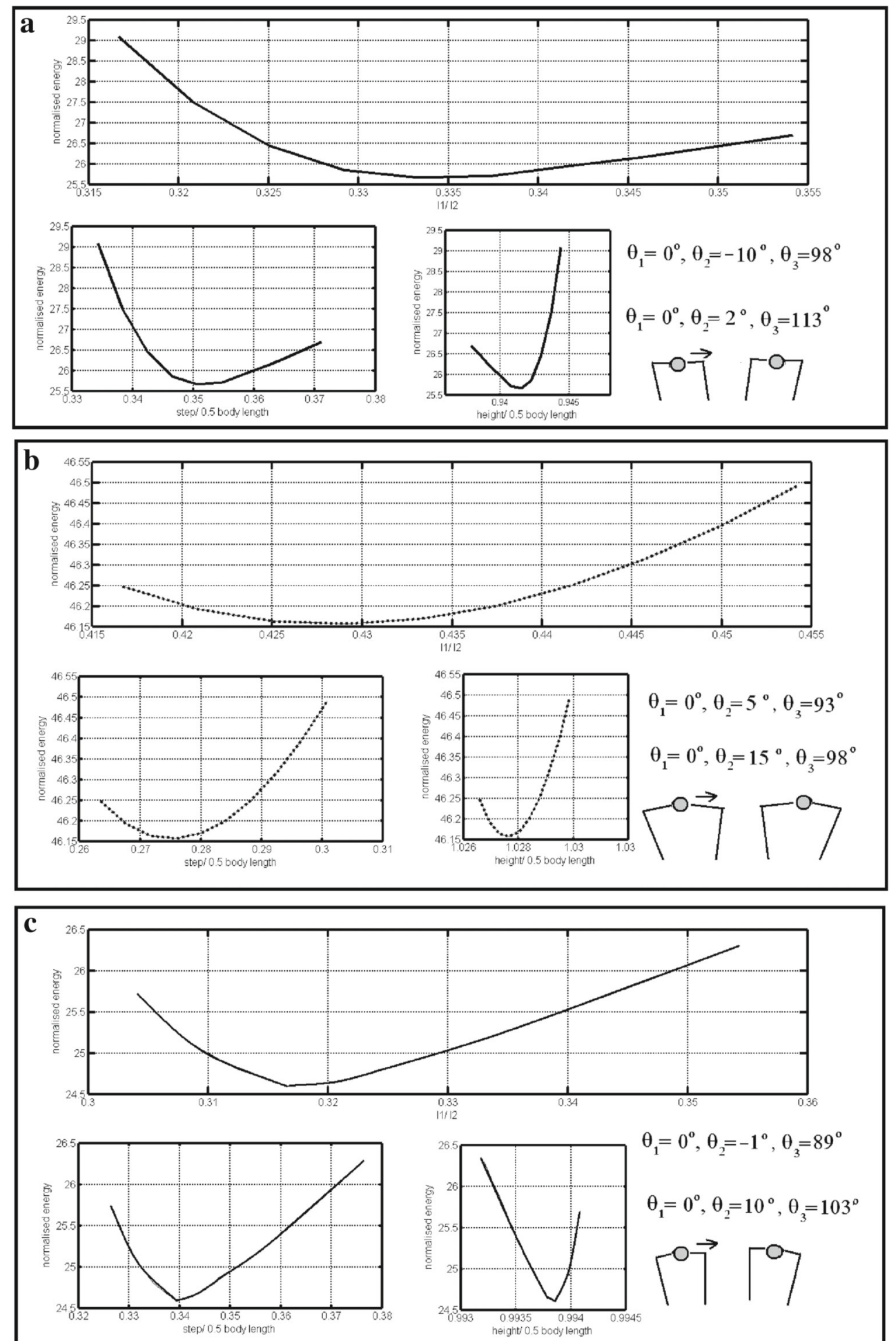

$\theta_{1}=0^{\circ}, \theta_{2}=-1^{\circ}, \theta_{3}=89^{\circ}$

$\theta_{1}=0^{\circ}, \theta_{2}=10^{\circ}, \theta_{3}=103^{\circ}$

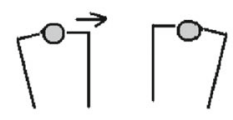

Fig. 10 Results for side walking: a - initial posture for legs seen on right side: $\theta_{1}=0^{\circ}, \theta_{2}=-10^{\circ}, \theta_{3}=98^{\circ}$, final: $\theta_{1}=0^{\circ}, \theta_{2}=2^{\circ}, \theta_{3}=113^{\circ}, \mathrm{b}-$ initial posture $\theta_{1}=3^{\circ}$, $\theta_{2}=5^{\circ}, \theta_{3}=93^{\circ}$, final: $\theta_{1}=0^{\circ}, \theta_{2}=15^{\circ}, \theta_{3}=98^{\circ}, \mathrm{c}-$ initial posture $\theta_{1}=0^{\circ}, \theta_{2}=-1^{\circ}, \theta_{3}=89^{\circ}$, final $\theta_{1}=0^{\circ}$, $\theta_{2}=-10^{\circ}, \theta_{3}=103^{\circ}$ 
Figure 8 illustrates the most representative results for straight line motion on a horizontal surface. Referring to our previous considerations, when selecting the most proper posture the step length and body height were considered also as important factors. Taking into account that the longest step produces the smallest number of leg transfers, for several postures having similar energy minimum the posture with the longest step should be selected. Another factor which must be considered is the body height, the greater the body height the better the machine negotiates obstacles. For the posture $\theta_{1}=0^{\circ}, \theta_{2}=-5.2^{\circ}, \theta_{3}=98^{\circ}$ the consumed energy reaches minimum if the thigh becomes equal to 0.9 of the shank length (Fig. 8b). Similar result is for the posture $\theta_{1}=0^{\circ}, \theta_{2}=$ $-15^{\circ}, \theta_{3}=107^{\circ}$ (Fig. 8c) with $l 1 / l 2=0.93$ and with comparable step length, but with slightly smaller height of the body. Therefore the posture $\theta_{1}=0^{\circ}$, $\theta_{2}=-5.2^{\circ}, \theta_{3}=98^{\circ}$ should be selected. In the posture $\theta_{1}=0^{\circ}, \theta_{2}=5.7^{\circ}, \theta_{3}=93^{\circ}$ (Fig. 8b) the consumed energy is more than two times higher than for the previous postures, the thigh to shank proportion for the energy minimum is about 0.525 . It must be noted that the step length longer than half of the body length will cause for symmetrical ranges of the steps that the legs will collide. The leg-end position at the beginning of the support phase will collide with the leg-end in front of it, ending its support phase, and so on. Therefore in our analysis we neglected the solutions with normalized step length greater than one. Such range was marked by rectangles in Fig. 8a, c.

Figure 9 illustrates the results for uphill walking with a slope of $10^{\circ}$. The energy increases significantly,

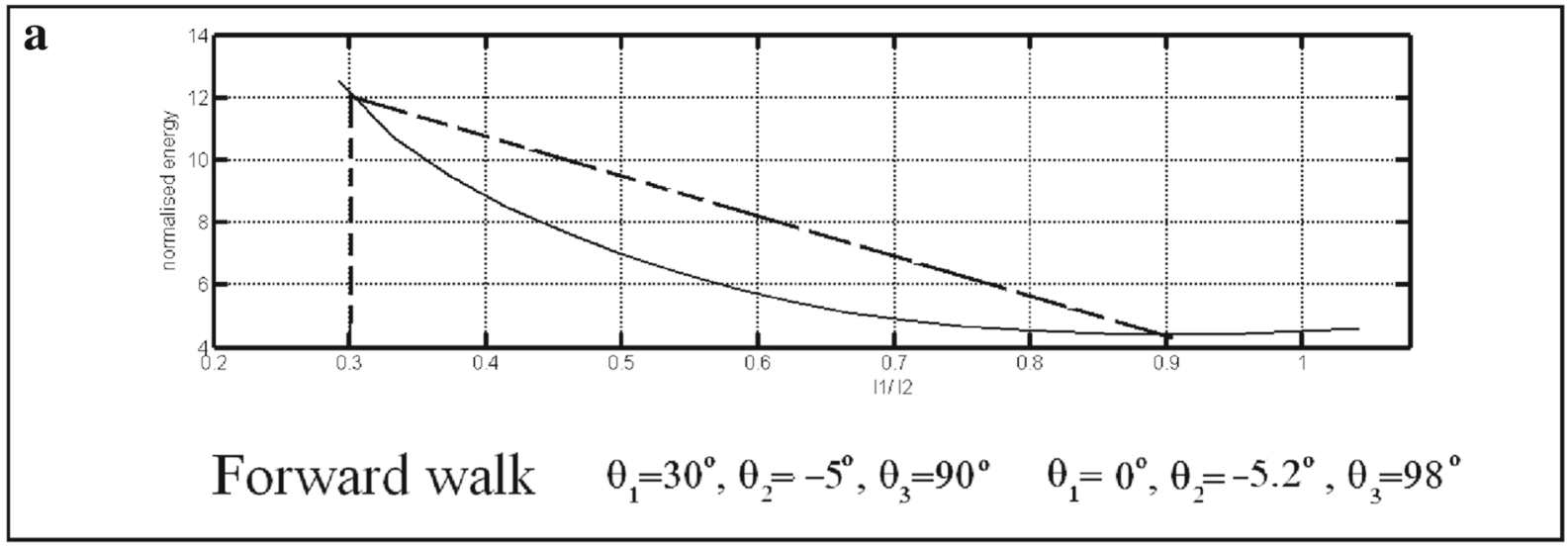

b

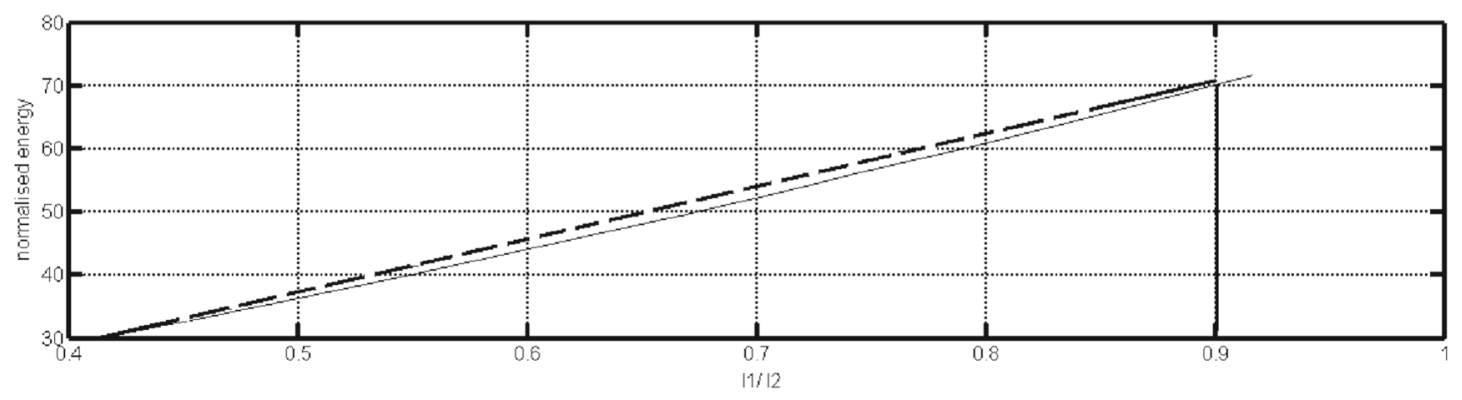

Side walk $\theta_{1}=0^{\circ}, \theta_{2}=-1^{\circ}, \theta_{3}=89^{\circ} \quad \theta_{1}=0^{\circ}, \theta_{2}=10^{\circ}, \theta_{3}=103^{\circ}$

Fig. 11 Energy demand during regular (a) and side gait (b) 
but the localisation of its minimum depending on the leg proportion is very close to those for the horizontal surface. For the posture $\theta_{1}=0^{\circ}, \theta_{2}=5.7^{\circ}$, $\theta_{3}=93^{\circ}$ it is $l 1 / l 2=0.55$ (Fig. 9a) versus 0.52 on the horizontal surface, and for posture $\theta_{1}=0^{\circ}$, $\theta_{2}=-5.7^{\circ}, \theta_{3}=98^{\circ}$ it is $l 1 / l 2=0.85(0.9)$. Increase of the terrain slope only slightly affects the leg proportion versus the energy minima, therefore it can be concluded that the leg proportions selected for the motion on horizontal surface are appropriate also for the motion on an inclined surface.

The last test concerned the side walking using a tripod gait. Figure 10 presents the selected results (walking to the right from the observer's point of view). The $x$ coordinate of the leg-end at the beginning of the step resulted from the posture and the length of the leg links, the step ended when the leg ends on the right of the body (observer's view) attained the level of the hip $x^{H}=0$. The minimum energy was achieved with the posture: $\theta_{1}=0^{\circ}, \theta_{2}=-1^{\circ}, \theta_{3}=89^{\circ}$ (starting position - legs seen on right side) $\theta_{1}=0^{\circ}$, $\theta_{2}=10^{\circ}, \theta_{3}=103^{\circ}$ (final position). For the legs on the left side the initial and final posture are interchanged. The optimal link proportion is close to 0.32 (Fig. 10c). Similar energy demand was obtained for the initial posture $\theta_{1}=0^{\circ}, \theta_{2}=-10^{\circ}, \theta_{3}=98^{\circ}$ (Fig. 10a). For the initial posture $\theta_{1}=0^{\circ}, \theta_{2}=5^{\circ}$, $\theta_{3}=93^{\circ}$ (Fig. 10b) $(l 1 / l 2=0.43)$ the consumed energy was about 1.8 times greater.

Favorable leg proportion, from the point of view of energy consumption, for side walking is very different than for regular walking, therefore if the mission of the machine requires both types of gaits the leg proportion must be selected accordingly.

Analyzing Fig. 11a it can be noticed that changing the optimum leg proportion $l 1 / l 2=0.9$ for the regular gait to 0.32 (side walking) increases the energy demand 3 times, from 4 to 12 units. Approximating the energy curve by straight line we obtain that with a decrease of leg proportions by 0.1 the energy increases by 1.3 . On the other hand for the side walking (Fig. 11b) the energy increases much faster and almost linearly with the increase of leg proportion. Increase of the proportion by 0.1 results in the energy increase by 8 units. Moreover, the energy demand in side walking is significantly higher than for the regular gait. This suggests that side walking should be used only occasionally.

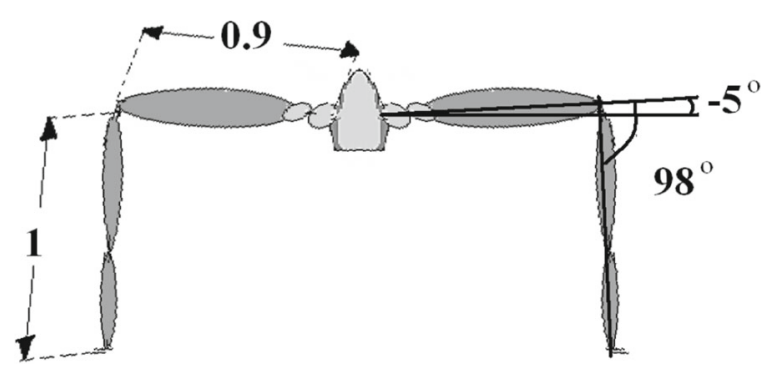

Fig. 12 Posture assuring the longest walking step and small energy consumption

This brings us to the final conclusion that for the long term missions the posture as considered in Fig. 8a and illustrated in Fig. 12 is the most appropriate.

As it was mentioned, changing the leg masses or the body proportions parameter does not change the localization of the energy minimum in relation to the leg segment proportions. So is the case for changing the motion speed.

\section{Conclusions}

The study of energy consumption depending on posture and leg proportion has been presented. The obtained results indicate that there exist leg proportions which are most favorable from the point of view of energy savings. The thigh length should be of about 0.9 of the shank length. We assumed that the leg mass was concentrated in the knee. The change of leg mass concentration will change the body dynamics what will slightly influence the results. The presented method of energy consumption analysis is useful when designing walking machines used for inspection or exploration. Such machines must be reliable and possibly simple, therefore they use identical legs, which are easy to assembly and control. Those machines must act autonomously, with on-board power supply, for a relatively long time, what still causes problems. Despite of better mobility than that of wheeled robots, walking machines due to their complexity and high power consumption are not often applied in special missions. The mentioned in the introduction Legged Squad Support System uses combustion engines that are noisy, air polluting and rather inefficient. The quest for silent and efficient legged robots is still going on. 
Open Access This article is distributed under the terms of the Creative Commons Attribution 4.0 International License (http:// creativecommons.org/licenses/by/4.0/), which permits unrestricted use, distribution, and reproduction in any medium, provided you give appropriate credit to the original author(s) and the source, provide a link to the Creative Commons license, and indicate if changes were made.

\section{References}

1. Burrows, M., Sutton, G.P.: The effect of leg length on jumping performance of short- and long-legged leafhopper insects. J. Exp. Biol. 211, 1317-1325 (2008)

2. Doroftei, I., Baudoin, Y.: A concept of walking robot for humanitarian demining. Ind. Robot. Int. J. 39(5), 441-449 (2012)

3. Erden, M.S., Leblebicioglu, K.: Analysis of wave gaits for energy efficiency. Auton. Robot. 23(3), 213-230 (2007)

4. Full, R.J., Ahn, A.N.: Static forces and moments generated in the insect leg: Comparision of a three-dimensional musco-sceletal computer model with experimental measurements. J. Exp. Biol. 198 (1995)

5. Garcia, E., Estremera, J., de Santos, G.P.: A control architecture for humanitarian-demining legged robots. In: Muscato, G., Longo, D. (eds.) Climbing and Walking Robots and the Supporting Technologies for Mobile Machines, pp. 383-390. Wiley (2003)

6. IFR Statistical Department: Executive Summary. World Robotics 2013: Service Robots, IFR (2014)

7. Jun, B.H., Shim, H., Kim, B.: Hexapod robot crabster CR200 for high tide, turbid water exploration six-legged robot built for stability, mobility in harsh environments. Sea Technol. 54(10), 33-40 (2013)

8. Kajita, S., Espiau, B.: Chapter: Legged robots. In: Siciliano, B., Khatib, O. (eds.) Handbook of Robotics, p. 2008. Springer

9. Kiguchi, K., Kusumoto, Y., Watanabe, K., Izumi, K., Fukuda, T.: Energy-optimal gait analysis of quadruped robots. Artif. Life Robot. 6(3), 120-125 (2002)

10. Kim, J.H.: Balance Stability and Energy Efficiency: Towards the Analogy between Robotic and Human Gait through Unified Models. IEEE Humanoids, pp. 1-2 (2013)

11. Kumar, V.R., Waldron, K.J.: Force distribution in closed kinematic chains. IEEE J. Robot. Autom. 4(6), 657-664 (1998)

12. Machado, J.A.T., Silva, M.F.: An Overview of Legged Robots. http://citeseerx.ist.psu.edu/viewdoc/summary? doi=10.1.1.106.8192 (2016)

13. Masum Billah, B., Mohiuddin, A., Soheli Farhana, F.: Walking hexapod robot in disaster recovery: developing algorithm for terrain negotiation and navigation. Int. J. Mech. Aerosp. Ind. Mechatron. Manuf. Eng. 2(6), 795-800 (2008)

14. Mahfoudi, C., Djouani, C., Rechak, S., Bouaziz, M.: Optimal force distribution for the legs of an hexapod robot. In:
Proceedings of 2003 IEEE Conference on Control Applications, pp. 657-663 (2003)

15. Marhefka, D.W., Orin, D.E.: Gait planning for energy efficiency in walking machines. Robotics and Automation. In: Proceedings of IEEE International Conference in Robotics and Automation, pp. 474-480 (1997)

16. Mena, L., Montes, H., Fernandez, R., Saria, J., Armada, M.: Reconfiguration of a climbing robot in all terrain hexapod robot. In: Robotics 2015: II-nd Iberian Robotics Conference. Advances in Robotics, vol. 2, pp. 197-208. Springer (2016)

17. Montes, H., Mena, L., Roemi, F., Sarria, J., Gonzalezde-Santos, P., Armada, M.: Hexapod robot for humanitarian demining. RISE 2015. In: 8th IARP Workshop on Robotics for Risky Environments (2015). http://hdl.handle. net/10261/111656

18. Nishii, J., Ogawa, K., Suzuki, R.: The optimal gait pattern in hexapods based on energetic efficiency. In: Proceedings of 3rd International Symposium on Artificial Life and Robotics, pp. 106-109. The Company of Biologists Limited 1995 (1996)

19. Nonami, K., Barai, R.K., Irawan, A., Daud, M.R.: Historical and Modern perspective of walking robots. In: Hydraulically Powered Hexapod Robots: Design, Implementation and Control, pp. 19-40. Springer (2014)

20. Pan, Y., Gao, F., Qi, C., Hai, X.C.: Human-tracking strategies for a six-legged rescue robot based on distance and view. Chin. J. Mech. Eng. 29(1), 1-12 (2016). http://www. cnki.net/kcms/detail/11.2737.TH.20151216.1405.008.html

21. Peng, S., Ding, X., Ceccarelli, M., Yang, F.: Force distribution based on loading capacity of a hexapod robot with insect-mammal mixed tripod gait. In: The 14th IFToMM World Congress. Taipei (2015). IFToMM.14TH.WC.OS13. 044

22. Popovic, M.B., Herr, H.: Ground Reference Points in Legged Locomotion: Definitions, Biological Trajectories and Control Implications. Mobile Robots Towards New Applications, ISBN 3-86611-314-5, Edited by A. Lazinica, pp. 79-104, ARS/plV, Germany, 2006. Open Access Database www.i-techonline.com

23. Roy, S.hibendu.S.hekhar., Pratihar, D.K.: Effects of turning gait parameters on energy consumption and stability of a six-legged walking robot. Auton. Robot. 72-82 (2012)

24. Roy, S.hibendu.S.hekhar., Pratihar, D.K.: Dynamic modeling, stability and energy consumption analysis of a realistic six-legged walking robot. Robot. Comput. Integr. Manuf. 29, 400-416 (2013)

25. Santos, P.G., Ponticelli, E.G., Armada, M.: Minimizing energy consumption in hexapod robot. Adv. Robot. 681704 (2009)

26. Sanz-Merodio, D., Garcia, E., Gonzalez-de-Santos, P.: Analyzing energy-efficient configurations in hexapod robots for demining applications. Ind. Robot. Int. J. 39(4), 357-364 (2012)

27. Vukobratovic, M., Borovac, B.: Zero moment point -35 years of its life. Int. J. Humanoid Rob. 1(1), 157-173 (2004)

28. Waldron, K.J., et al.: Configuration design of the adaptive suspension vehicle. Int. J. Robot. Res. 3(2), 3748 (1984) 
29. Zielinska, T., Heng, J.: Real-time control system for a group of autonomous walking robots. Adv. Robot. 20(5), 543-561 (2006)

30. Zielinska, T.: Autonomous walking machines - discussion of prototyping problems. Bull. Pol. Acad. Sci. 58(3), 443451 (2010)

31. Zielinska, T.: Efficiency analysis in the design of walking machines. J. Theor. Appl. Mech. 3(38), 693-708 (2000)

32. Zielinska, T., Heng, J.: Development of Walking machine: mechanical design and control problems. Mechatron. Int. J. 12, 737-754 (2002). Elsevier, Mechatronics
Teresa Zielinska is full professor at Warsaw University of Technology, Faculty of Power and Aerospace Engineering,. She conducts her research work in the area of: design and control of robotic devices, methods of recognizing the surrounding using diverse sensors, principles governing mobile robots cooperation, novel design and control solutions for humanoids and walking machines. Her research interest focuses on autonomous devices with enhanced mobility and autonomy. She designed and implemented the quadruped walking machine control system and control systems for motion control and navigation for the six-legged machines performing autonomously the group tasks. She is the Senior Member of IEEE and Secretary General of IFToMM, she is also the local chair of European Master on Advanced Robotics (EMARO+) the prestigious international master program supported by ERASMUS+ funds. 